\title{
Hierarchical Incorporation of Surface-Functionalized Laponite Clay Nanoplatelets with Type I Collagen Matrix
}

\author{
Jiabo Shi,,${ }^{1 *}$ Ruizhen Zhang, ${ }^{1} \mathrm{Na}$ Yang, ${ }^{1}$ Yi Zhang, ${ }^{2}$ Bradley W. Mansel, ${ }^{3}$ Sujay Prabakar, ${ }^{2}$ \\ and Jianzhong $\mathrm{Ma}^{1, *}$ \\ ${ }^{1}$ College of Bioresources Chemical and Materials Engineering and National Demonstration Center for \\ Experimental Light Chemistry Engineering Education, Shaanxi University of Science \& Technology, No.6 \\ Xuefu Zhonglu, Weiyang District, Xi'an, 710021, China \\ ${ }^{2}$ Leather and Shoe Research Association of New Zealand, P.O. Box 8094, Palmerston North 4472, New \\ Zealand \\ ${ }^{3}$ Chemical Engineering Building, National Tsing Hua University, No. 101, Section 2, Guangfu Road, East \\ District, Hsinchu City, 300 Taiwan, China
}

\section{Corresponding Authors:}

1*E-mail: shijiabo@sust.edu.cn; ${ }^{2 *} E$-mail: majz@sust.edu.cn

Figure S1. Appearance and 2D surface micrograph of cross-section of pristine bovine collagen fibers (a) before and (b) after incorporated with LAP-THPS NPs: (a', a',) true and (b', b') pseudo color.

Figure S2. 3D CLSM image of LAP-THPS-CFs nanocomposites incorporated with rhodamine $6 \mathrm{G}$-stained NPs. The red fluorescence shows the binding sites of the NPs within the CFs matrix. Figure S3. EDX elemental mappings of LAP-THPS-CFs nanocomposites showing simultaneous presence of elements of (a) carbon ( $\mathrm{C}, \mathrm{red}),(\mathrm{b})$ nitrogen $(\mathrm{N}$, yellow), (c) silicon ( $\mathrm{Si}$, green) and (d) magnesium (Mg, purple).

Figure S4. AFM images of collagen fibrils of pristine CFs matrix. Yellow arrow shows typical $D$-period banding patterns.

Figure S5. DSC thermographs of pristine CFs and LAP-THPS-CFs nanocomposites incorporated with $2 \%$ and $4 \%$ LAP-THPS NPs. The corresponding temperature of denaturation, denoted as $T_{\mathrm{d}}$, are determined to be $82.5^{\circ} \mathrm{C}, 88.2^{\circ} \mathrm{C}$ and $93.6{ }^{\circ} \mathrm{C}$, respectively.

Figure S6. (a) Storage modulus $E^{\prime}$, (b) stress-strain curves, (c) tensile strength, and (d) elongation of pristine CFs, LAP-CFs, and LAP-THPS-CFs nanocomposites incorporated with $4 \%$ NPs.

Figure S7. Amino acid analysis of bovine collagen fibers (a) before and (b) after incorporated with LAP-THPS NPs.

Figure S8. (a) XPS wide scan spectra and (b) enlarged XPS spectra between 0-250 eV of pristine CFs and LAP-THPS-CFs, and the corresponding high-resolution XPS spectra of (c) O 1s and (d) N 1s core level. 

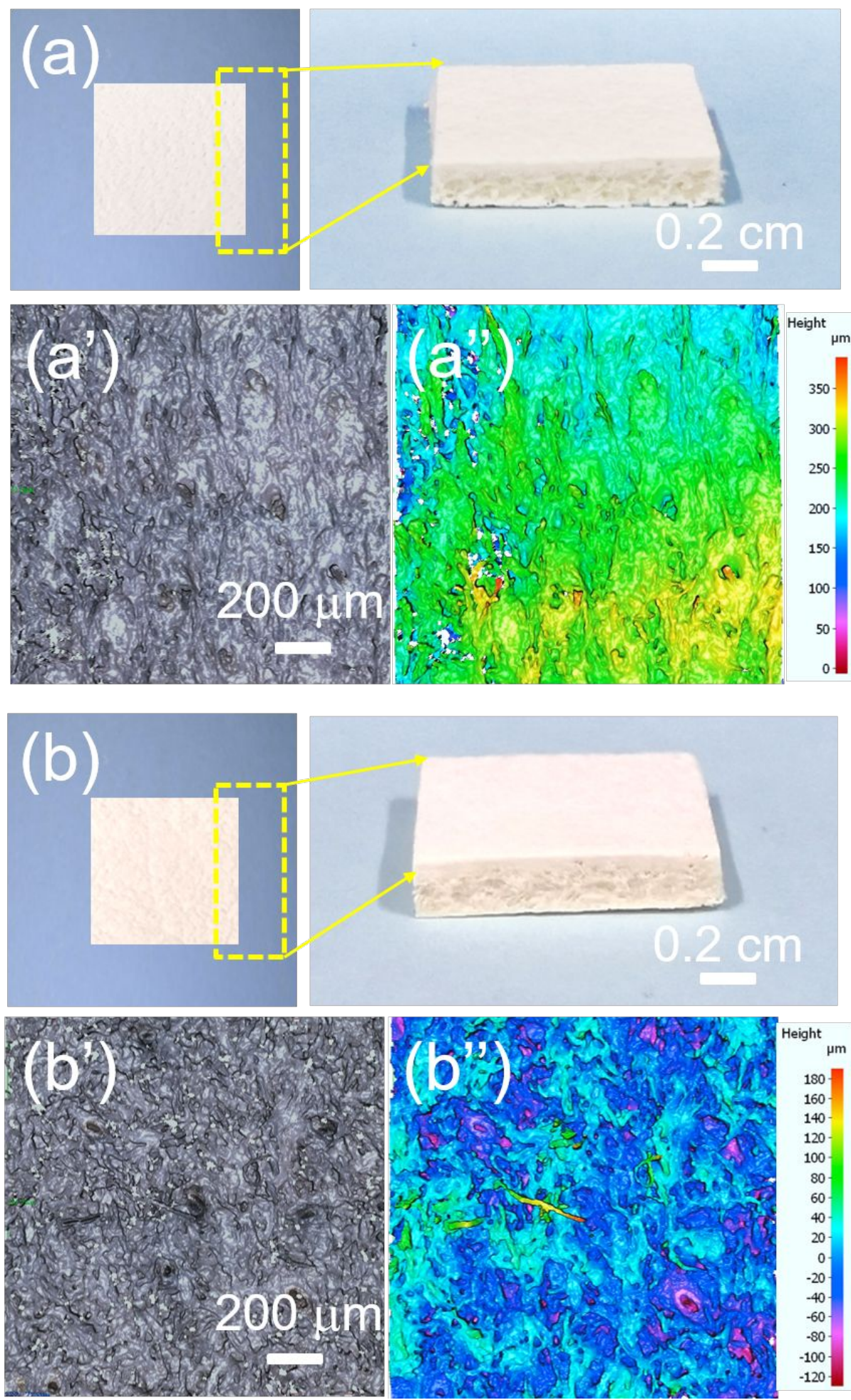

Figure S1. Appearance and 2D surface micrograph of cross-section of pristine bovine collagen fibers (a) before and (b) after incorporated with LAP-THPS NPs: (a', a') true and (b', b') pseudo color. 


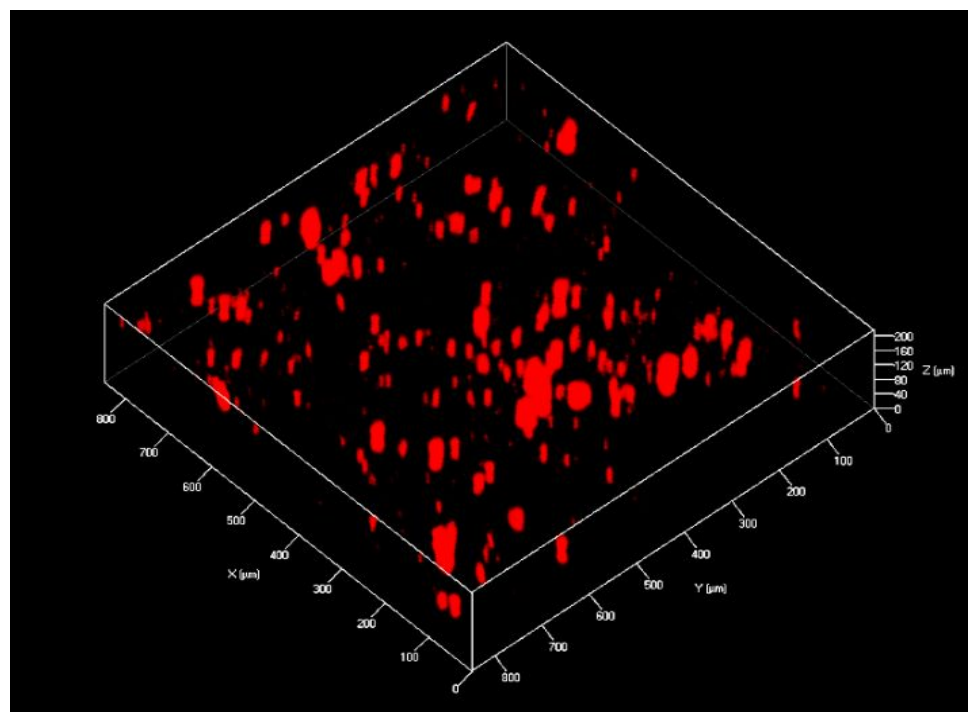

Figure S2. 3D CLSM image of LAP-THPS-CFs nanocomposites incorporated with rhodamine 6G-stained NPs. The red fluorescence shows the binding sites of the NPs within the CFs matrix.
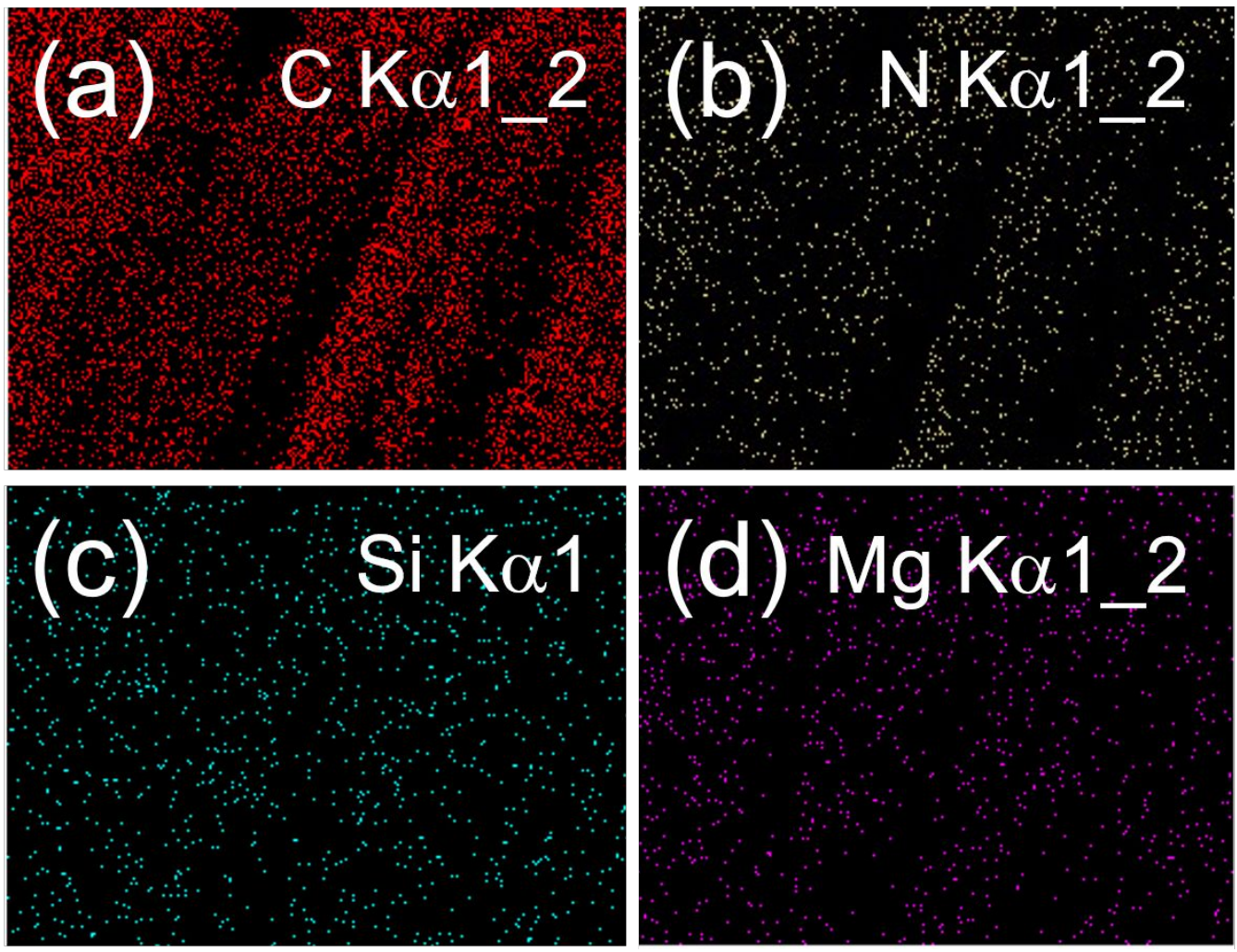

Figure S3. EDX elemental mappings of LAP-THPS-CFs nanocomposites showing simultaneous presence of elements of (a) carbon (C, red), (b) nitrogen (N, yellow), (c) silicon ( $\mathrm{Si}$, green) and (d) magnesium ( $\mathrm{Mg}$, purple). 

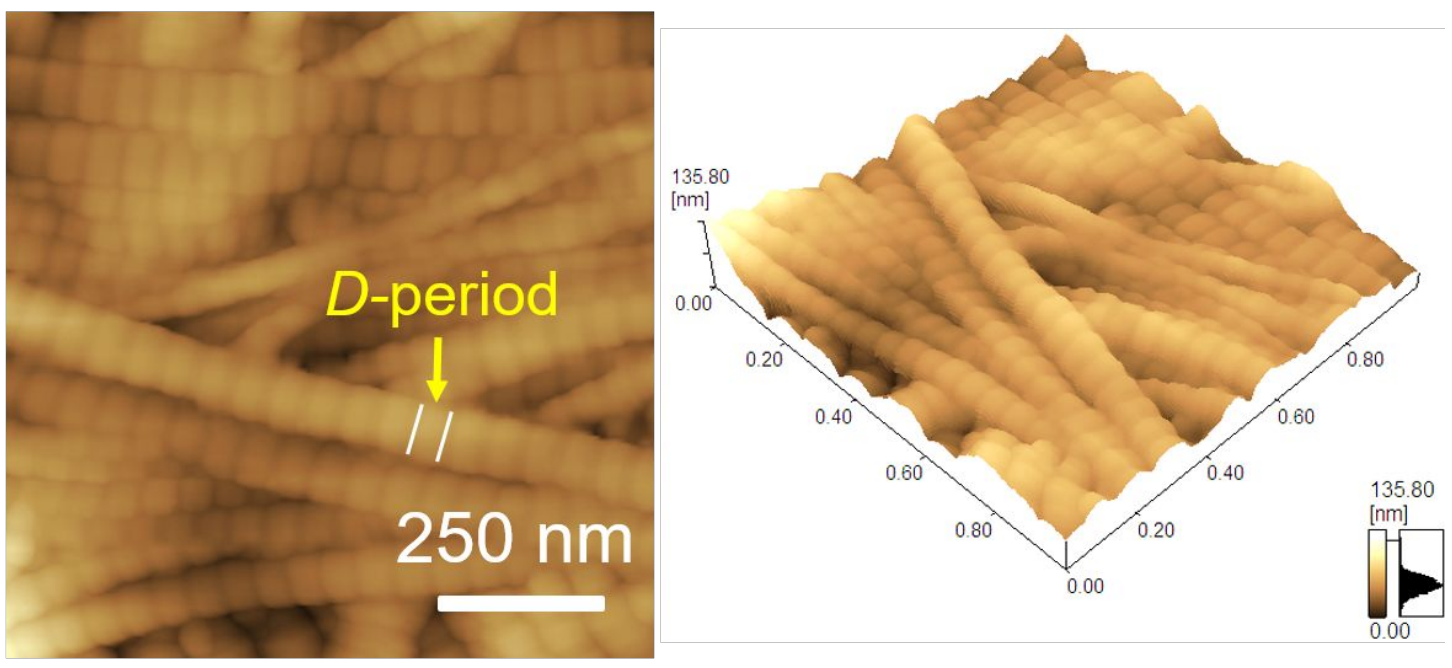

Figure S4. AFM images of collagen fibrils of pristine CFs matrix. Yellow arrow shows typical $D$-period banding patterns.

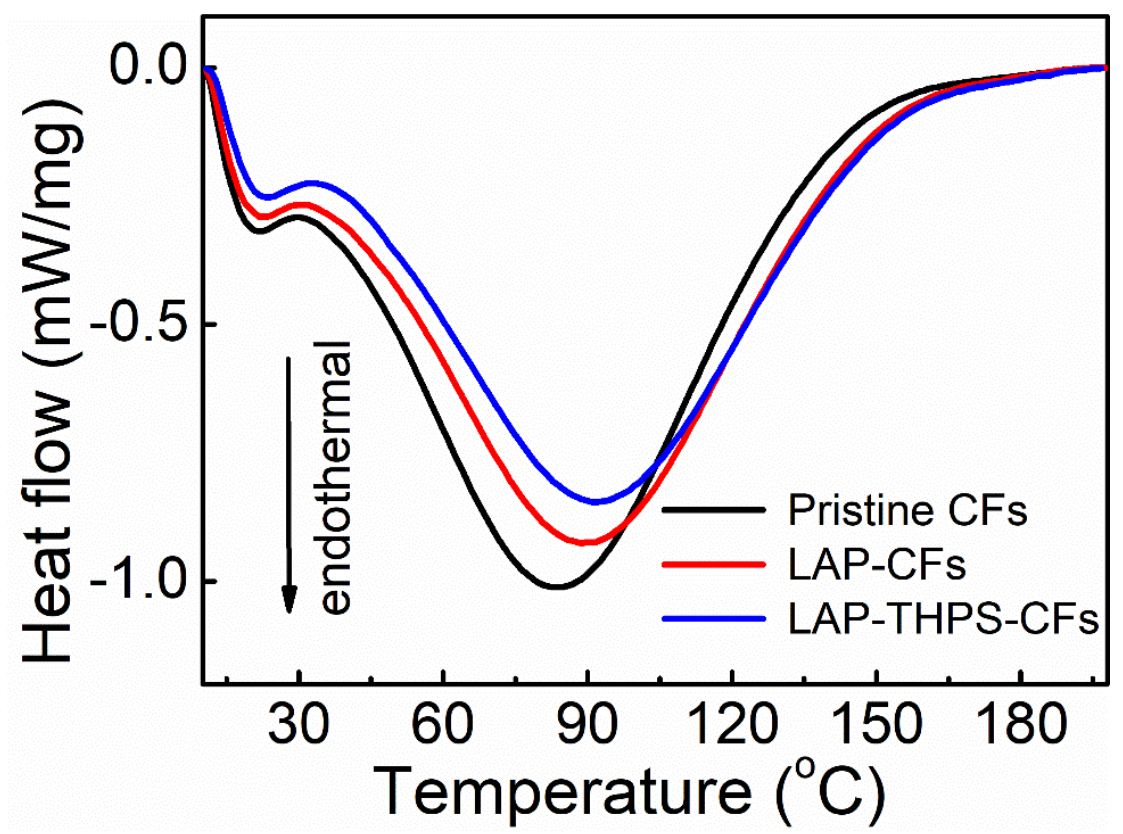

Figure S5. DSC thermographs of pristine CFs, LAP-CFs and LAP-THPS-CFs nanocomposites incorporated with $4 \%$ NPs. The corresponding temperature of denaturation, denoted as $T_{\mathrm{d}}$, are determined to be $82.5^{\circ} \mathrm{C}, 88.2^{\circ} \mathrm{C}$ and $93.6^{\circ} \mathrm{C}$, respectively. 

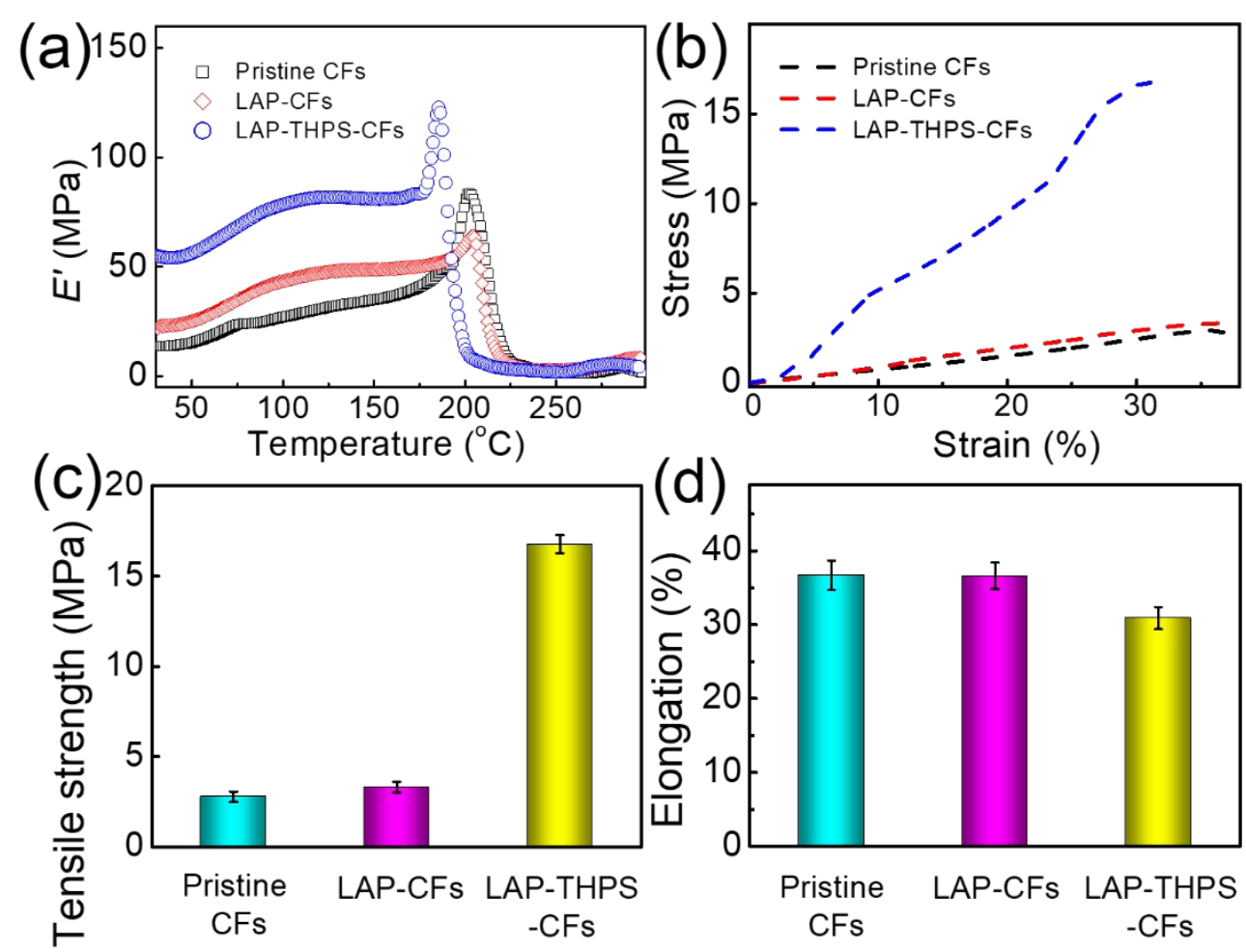

Figure S6. (a) Storage modulus E', (b) stress-strain curves, (c) tensile strength, and (d) elongation of pristine CFs, LAP-CFs, and LAP-THPS-CFs nanocomposites incorporated with $4 \%$ NPs.

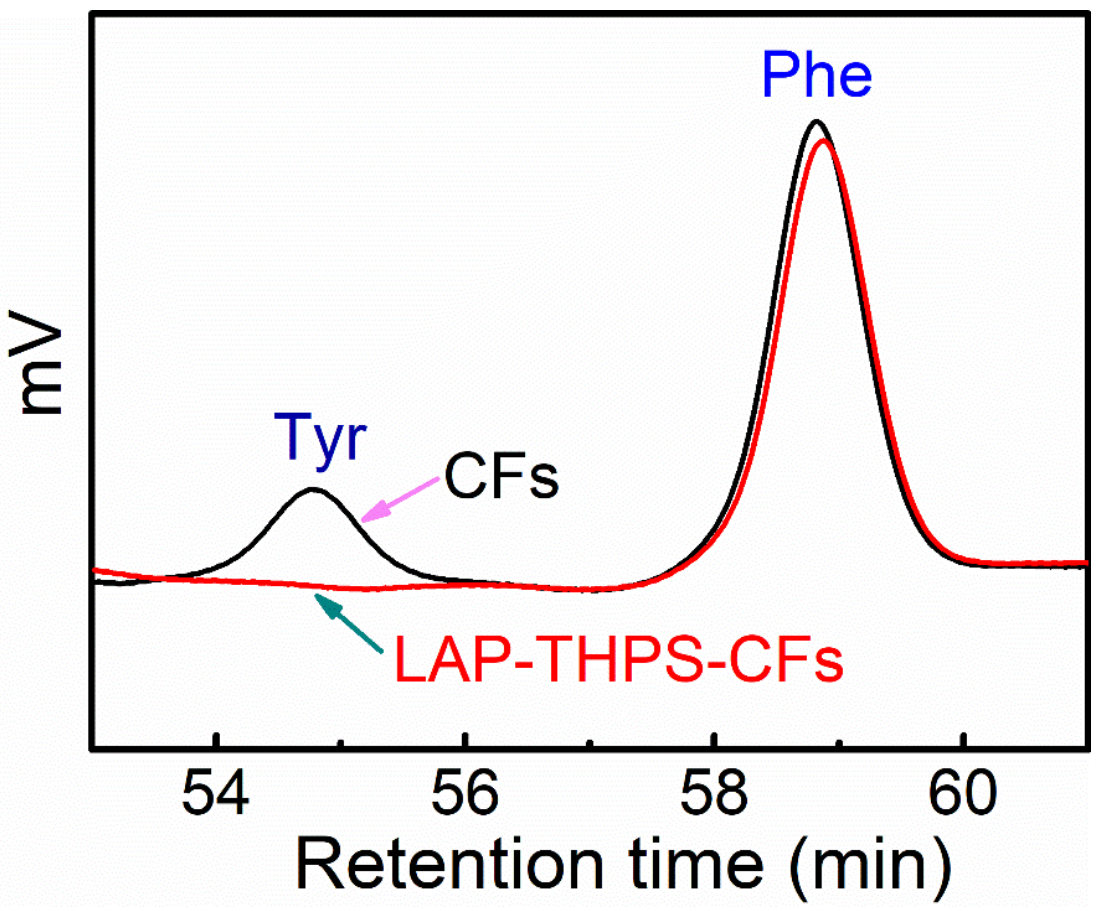

Figure S7. Amino acid analysis of bovine collagen fibers (a) before and (b) after incorporated with LAP-THPS NPs. 

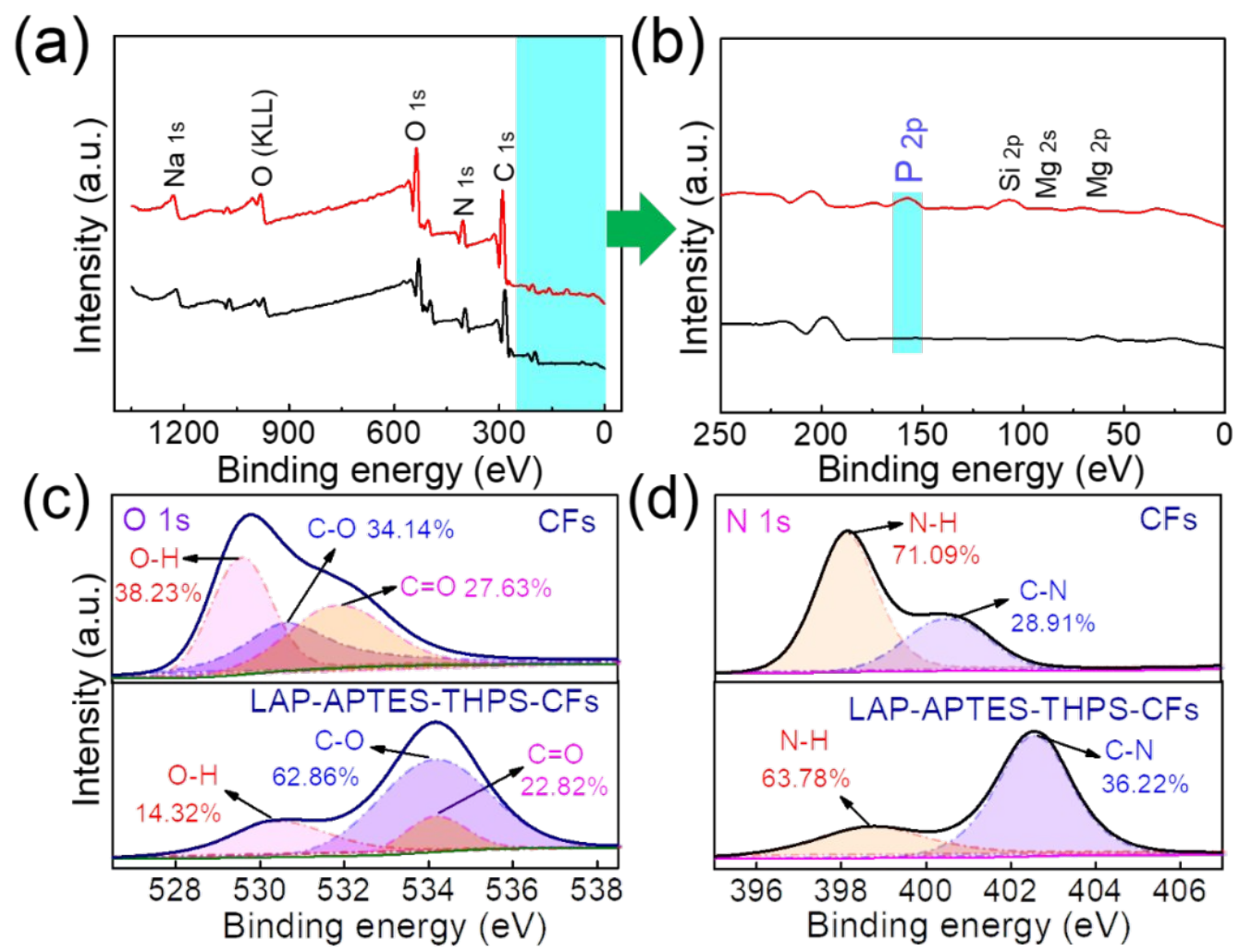

Figure S8. (a) XPS wide scan spectra and (b) enlarged XPS spectra between 0-250 eV of pristine CFs and LAP-THPS-CFs, and the corresponding high-resolution XPS spectra of (c) $\mathrm{O}$ 1s and (d) $\mathrm{N}$ 1s core level. 